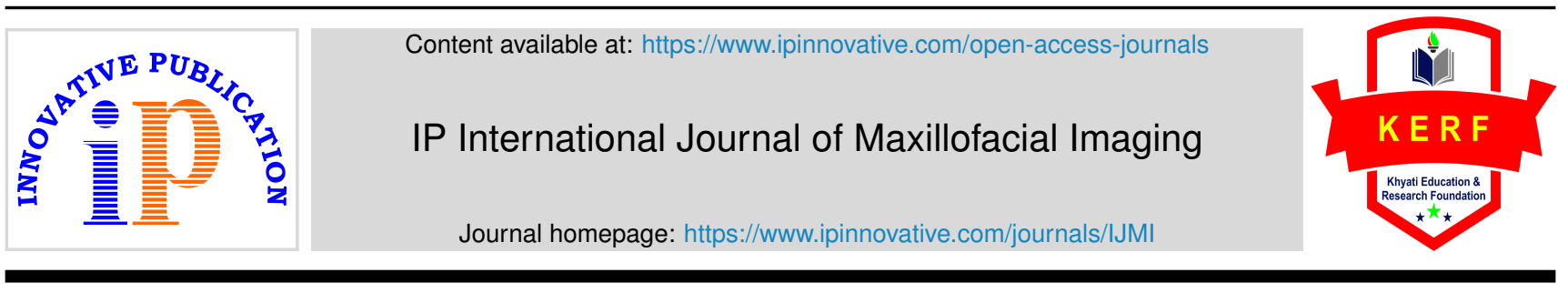

Original Research Article

\title{
Prevalence of mesiobuccal 2 canal in first maxillary molar: A retrospective radiographic study
}

\author{
Altaf Hussain Chalkoo', Rayees Ahmad Sheikh ${ }^{1}$,*, Shamshad Begum ${ }^{1}$ \\ ${ }^{1}$ Dept. of Oral Medicine and Radiology, Government Dental College and Hospital, Srinagar, Jammu \& Kashmir, India
}

\section{A R T I C L E I N F O}

\section{Article history:}

Received 28-11-2020

Accepted 04-12-2020

Available online 15-01-2021

\section{Keywords:}

CBCT

Mesiobuccal

Canal

First molar

\begin{abstract}
A B S T R A C T
Finding two mesiobuccal root canals named MB1and MB2 in maxillary first molar is not uncommon. Mesio-buccal-2 (MB2) canal orifice located palatally to the mesio buccall orifice and buccally as well as mesially to palatal orifices. A complete understanding of MB2 root canal morphology can contribute to the successful outcome of root canal treatment. This study conducted on CBCT scans with age ranged from 15 to 60 years which was further divide into group I (15-30 years), group II (30-45 years) and group III (45-60 years). Unilateral as well as bilateral prevalence of MB2 canal in maxillary first molar in both the genders and prevalence of MB2 canal in maxillary first molar in accordance with age were determined. Results showed overall prevalence of MB2 canal in maxillary first molar was $60.7 \%$. In age wise distribution, group II showed greater prevalence than group I and group III showed least prevalence than others. The results were statistically significant between age groups. There was no statistical significance obtained between gender and quadrant wise. Conventional diagnostic aids such as radiographs play an important role in assessment of complex root canal morphologies. These modalities, however, do not provide detailed information of the complexity as a result of their inherent limitations. Shifting from 2D radiographic techniques to $3 \mathrm{D}$ radiographic techniques provides better visualization and location of these small and difficult structures.
\end{abstract}

(C) This is an open access article distributed under the terms of the Creative Commons Attribution License (https://creativecommons.org/licenses/by/4.0/) which permits unrestricted use, distribution, and reproduction in any medium, provided the original author and source are credited.

\section{Introduction}

Root canal therapy is one of the routinely carried out procedure in dentistry applicable on teeth with irreversible pulpitis and periapical pathologies in order to preserve the natural dentition. A detailed knowledge and awareness of complex anatomy of the root canals is utmost important for success of endodontic treatment. ${ }^{1}$ Amongst all the causes of root canal treatment failures for example inadequate canal debridement due to anatomical complexities and canal preparation, the failure to locate the accessory canals also leads to unfavorable treatment.

The maxillary first molar teeth usually possess a great anatomical variation in terms of number of root canals, size and shape of canal. Very often an accessory root canal is

\footnotetext{
* Corresponding author.

E-mail address: shkrays@gmail.com (R. A. Sheikh).
}

present lingual/palatal to the mesiobuccal canal of theses molar, which is commonly not negotiated by practioner may further lead to root canal treatment failure. This canal is known as the mesiolingual canal of the mesiobuccal root, mesiopalatal canal, or MB2. ${ }^{2}$ The presence of such root canals was first reported by Hess in $1925 .{ }^{3}$ Christie WH (1991) and Fogel HM (2017) reported variation in distobuccal root canal and palatal canals of maxillary molars. ${ }^{4,5}$ According to Witherspoon DE (2013), $44 \%$ of retreatment in maxillary first molars is due to missed canals and $93 \%$ of these missed canals are identified in the mesiobuccal-2 root canal. ${ }^{6}$

Cone beam computed tomography (CBCT) imaging is an invaluable radiographic modality which shows the root canal system in three dimensions with superior contrast and resolution compared to $2 \mathrm{D}$ radiographic techniques. It provides better understanding of anatomical complexities 
and guides the clinician to more efficient treatment procedures.

\section{Aim}

The aim of this retrospective radiographic study is to estimate the prevalence of MB2 canal in maxillary first molar in both the gender and in different age groups using cone beam computed tomography (CBCT) images in North Indian population.

\section{Objectives}

The objective of this study is to investigate the following features using Cone Beam Computerized Tomography:-

1. Unilateral as well as bilateral prevalence of MB2 canal in maxillary first molar in both the genders.

2. Prevalence of MB2 canal in maxillary first molar in accordance with age.

\section{Materials and Methods}

This retrospective radiographic study conducted on 135 patients (73 males and 62 females). Their CBCT scans were obtained by Kodak CS 9300 digital imaging system (Tube Focal spot-0.7mm, Tube Voltage- 60-90 Kvp Tube Current (mA)- 5-15mA). The age of patients ranged from 15 to 60 years which was further divide into group I (15-30 years), group II (30-45 years) and group III (45-60 years), they were subjected to various measurements in cognizance with objectives of the study.

The inclusion and exclusion criteria were defined as follows:

\subsection{Inclusion criteria}

Patients above the age of 15 years with healthy maxillary permanent first molar teeth on both sides. The maxillary first molars with complete root formation.

\subsection{Exclusion criteria}

Maxillary first molar with developmental anomalies, open apices, root fractures, internal or external resorption and pulp calcifications.

The floor of the pulp chamber of maxillary first molar was first determined in sagittal section. By using liner measurement tool of the CBCT software, a $2 \mathrm{~mm}$ line drawn from the floor towards apical end. The axial section $2 \mathrm{~mm}$ below the pulpal floor was taken as standardize observation point of the MB2 canal and presence or absence of MB2 canal was then noted in the axial view (Figures 1, 2 and 3). This geometric position was according to the protocol described by Gorduysus et al. ${ }^{7}$ and Betancourt et al. ${ }^{8}$ The data was correlated with age, gender and side. For suitable and appropriate statistical analysis, SPSS version 16.0 has been used. A 5\% level of significance has been chosen and the p-value of less than 0.05 has been considered as statistically significant and less than 0.001 has been considered as highly significant.

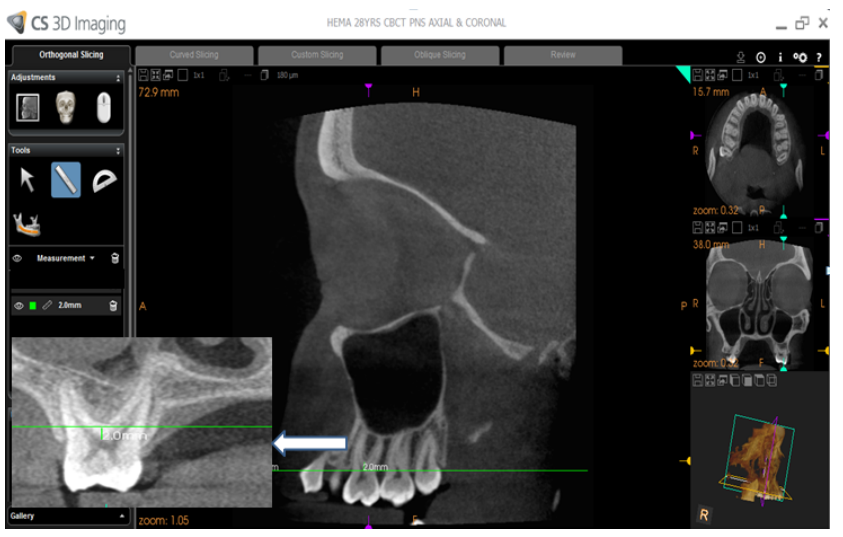

Fig. 1: Determination of $2 \mathrm{~mm}$ below the pulpal floor

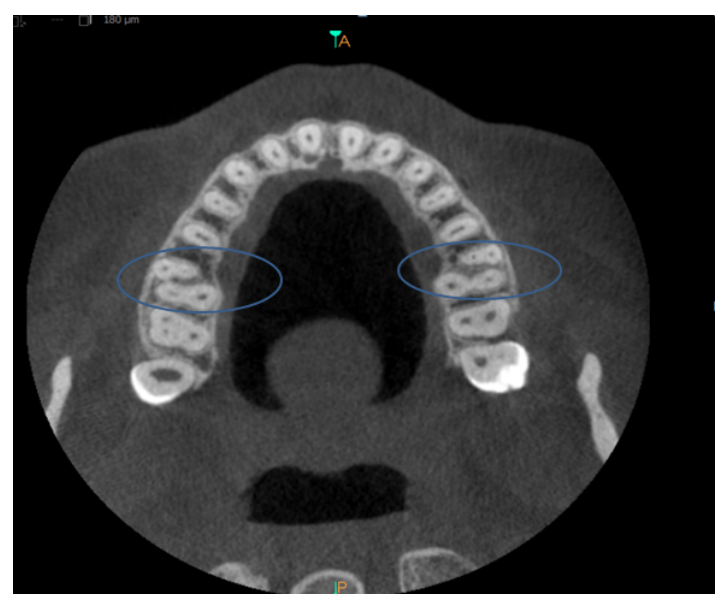

Fig. 2: Bilateral presence of MB2 canal in first maxillary molar

\section{Results}

This study showed the prevalence of MB2 canal in different age groups. Out of total 135 scans, 82 patients (45 males and 37 females) showed presence of MB2 canal in maxillary first molar. The overall prevalence of MB2 canal in maxillary first molar was $60.7 \%$. In age wise distribution, group I showed 57\%(22), group II showed 66\% (43) and group III showed 53\% (17) prevalence of MB2 canal (Table 1). There results were statistically significant between age groups.

Gender wise unilateral and bilateral presence of MB2 canal in maxillary first molar showed that in males, bilateral MB2 canal in 17(37.7\%) cases and unilateral in $28(62.2 \%)$ cases and in females, bilateral MB2 canal in $14(37.8 \%)$ cases and unilateral in $23(62.1 \%)$ cases. Side 
Table 1: Age wise distribution of presence of MB2 canal

\begin{tabular}{lcccccc}
\hline Age Group & $\begin{array}{c}\text { Group I } \\
(\mathbf{1 5 - 3 0 y r s )}\end{array}$ & $\begin{array}{c}\text { Group II } \\
\mathbf{( 3 0 - 4 5 y r s )}\end{array}$ & Group III (45-60yrs) & Total & $\chi^{2}$ & p-value \\
Total no & 38 & 65 & 43 & 32 & 135 & \\
Present MB2 & 22 & $66 \%$ & $53 \%$ & 82 & 29.18 & $0.0005^{*}$ \\
Percent Age & $57 \%$ & $60.7 \%$ & 17 & \\
\hline
\end{tabular}

Table 2: Gender wise unilateral/bilateral presence of MB2 canal in maxillary first molar

\begin{tabular}{|c|c|c|c|c|c|c|c|c|c|}
\hline \multirow[t]{2}{*}{ Gender } & \multirow[t]{2}{*}{ No. } & \multirow{2}{*}{$\begin{array}{c}\text { Bilateral } \\
\text { MB2 } \\
\text { canal }\end{array}$} & \multicolumn{5}{|c|}{ Unilateral MB2 canal } & \multirow[t]{2}{*}{$\chi^{2}$} & \multirow[t]{2}{*}{ p-value } \\
\hline & & & Right & Left & Total & $X^{2}$ & p-value & & \\
\hline \multirow[t]{2}{*}{ Male } & 45 & 17 & 15 & 13 & 28 & \multirow{2}{*}{0.14} & \multirow{2}{*}{0.97} & & \\
\hline & $54.8 \%$ & $37.7 \%$ & $33.3 \%$ & $28.8 \%$ & $62.2 \%$ & & & & \\
\hline \multirow[t]{2}{*}{ Female } & 37 & 14 & 12 & 11 & 23 & \multirow{2}{*}{0.04} & \multirow{2}{*}{0.97} & 0.48 & $>0.5$ \\
\hline & $45.2 \%$ & $37.8 \%$ & $32.4 \%$ & $29.7 \%$ & $62.1 \%$ & & & & \\
\hline \multirow[t]{2}{*}{ Total } & 82 & 31 & 27 & 24 & 51 & & & & \\
\hline & $100 \%$ & $37.8 \%$ & $53 \%$ & $47 \%$ & $62.2 \%$ & & & & \\
\hline
\end{tabular}

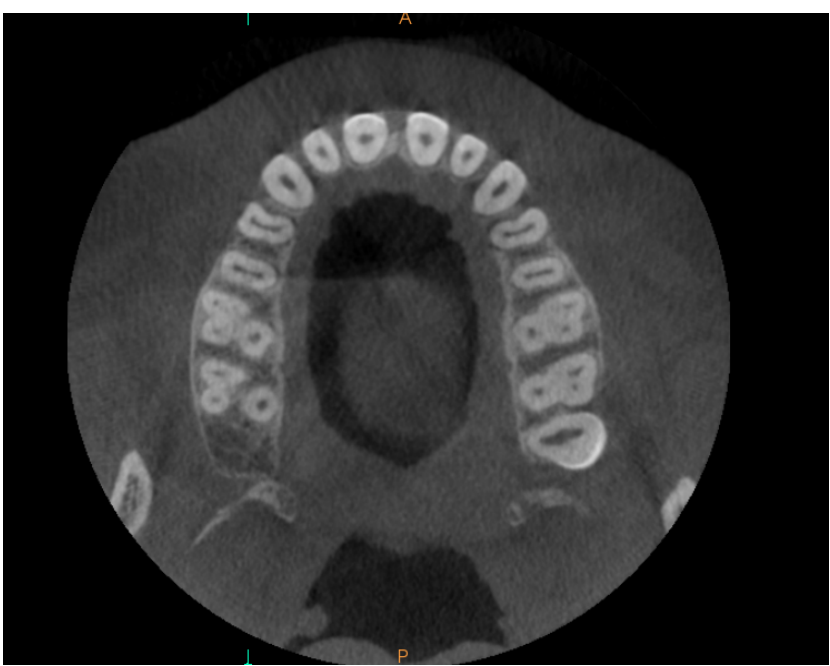

Fig. 3: Unilateral presence of MB2 in right first maxillary molar

wise distribution showed that right side had greater number of MB2 canal than the left side in both the gender. There were no statistical significance obtained between gender and quadrant wise.

\section{Discussion}

The results of our study indicated that CBCT helps clinician to better understand the morphological variations of root canal due to its high diagnostic accuracy and thereby increasing the chances of endodontic treatment success. Our study reported statistically significant results between age groups and the prevalence of MB2 canal in maxillary first molar was $60.7 \%$. The prevalence obtained by Kewalramani R (2019) ${ }^{9}$ was $61.9 \%$ which was similar to our study. In contrast, Neelakantan $\mathrm{P}$ et al. ${ }^{10}$ and Karunakar et al ${ }^{11}$ have reported low prevalence of MB2 in maxillary first molars in Indian population to be $44.1 \%$ and $47.1 \%$, respectively and Kashyap RR et al ${ }^{12}$ found MB2 canal in $76.5 \%$ of maxillary first molars which is higher than our results.

Age Group II showed high prevalence of MB2 canal than group I followed by age group III. The results were statistically significant. Similar results obtained by Kewalramani R $(2019)^{9}$ in which group $<20$ years $(50.6 \%)$ and $>40$ years $(57.5 \%)$ showed less prevalence compared to $20-40$ years $(67.4 \%)$. The low prevalence in age group I can be due to presence of single wide mesiobuccal orifice. In age group III, decreased prevalence was observed in our study and it may be due to obliteration of canal because of secondary dentine formation leading to dentinal sclerosis and pulpal recession. The results were also consistent with the studies of Reis et al. ${ }^{13}$ and Lee et al. ${ }^{14}$ Our study findings suggest that in patients within the 30-45 age group requires attention in negotiating MB2 canal by careful troughing and magnification but not beyond the furcation.

In our study, there were no statistical significance obtained between gender and quadrant wise, although side wise distribution showed that right side had greater number of MB2 canal than the left side in both the gender. The prevalence of MB2 canal was $92 \%$ for permanent first maxillary molar teeth in the first quadrant, $87 \%$ for permanent first maxillary molar teeth in the second quadrant in both gender in a study done by Fernandes NA (2019) ${ }^{15}$ and also did not show a significant association between patient gender. Similar studies in the literature showed same results. ${ }^{13,16}$

\section{Conclusion}

Root canal morphology is commonly assessed on periapical (PA) radiographs which is a $2 \mathrm{D}$ radiographic modality and hence provides inadequate visualization of presence 
of extra canals, such as MB2 which leads to incomplete treatment and symptoms of pulpal infection remains even after root canal treatment of the tooth. CBCT reveal more accurate internal anatomy of root canals especially MB2 canals which can be easily neglected by clinician in two dimensional radiography.

\section{Source of Funding}

None.

\section{Conflict of Interest}

The authors declare that there is no conflict of interest regarding the publication of this article.

\section{References}

1. Krasner P, Rankow H. Anatomy of the Pulp-Chamber Floor. J Endod. 2004;30(1):5-16. 10i:1010970000477(0-200401000(00002

2. Kulid JC, Peters DD. Incidence and configuration of canal systems in the mesiobuccal root of Maxillary first and second molars. J Endod. 1990;16(7):311-7. do1:10.1016/s0099-2399(06)81940-0.

3. Coelho MS, Lacerda M, Silva MHC, Rios MA. Locating the second mesiobuccal canal in maxillary molars: challenges and solutions. Clin, Cosmet Investig Dent. 2018;10:195-202. 101:102147/ccides S15464].

4. Christie WH, Peikoff MD, Fogel HM. Maxillary molars with two palatal roots: A retrospective clinical study. J Endod. 1991;17(2):80 4. ब01:10.1016/s0099-2399(06)81613-4

5. Fogel HM, Cunha RS. Maxillary First Molars with 2 Distobuccal Canals: A Case Series. $J$ Endod. 2017;43(11):1925-8. doi:10.1016/j.joen.2017.07.004

6. Witherspoon DE, Small JC, Regan JD. Missed canal systems are the most likely basis for endodontic retreatment of molars. Tex Dent J. 2013;130:127-39.

7. Görduysus MO, Görduysus M, Friedman S. Operating Microscope Improves Negotiation of Second Mesiobuccal Canals in Maxillary

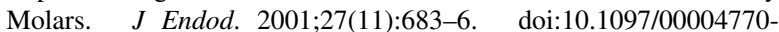
एवण्ण $000-00008$.

8. Betancourt P, Navarro P, Cantín M, Fuentes R. Cone-beam computed tomography study of prevalence and location of MB2 canal in the mesiobuccal root of the maxillary second molar. Int J Clin Exp Med. 2015;8:9128-34.

9. Kewalramani R, Murthy CS, Gupta R. The second mesiobuccal canal in three-rooted maxillary first molar of Karnataka Indian sub- populations: A cone-beam computed tomography study. J Oral Biol Craniofac Res. 2019;9(4):347-51. doi:10.1016/j.jobcr.2019.08.001.

10. Neelakantan P, Subbarao C, Ahuja R, Subbarao CV, Gutmann JL. Cone-Beam Computed Tomography Study of Root and Canal Morphology of Maxillary First and Second Molars in an Indian Population. $J$ Endod. 2010;36(10):1622-7. do1:10.1016/].joen.2010.07.006

11. Byragoni C, Sanjana L, Komali G, Karunakar P, Solomon RV. Demystifying the mesiobuccal root of maxillary first molar using cone-beam computed tomography. Indian J Dent Res. 2015;26(1):63. 10i:-10.4103/0970-9290.156810.

12. Kashyap RR, Rao PK, Beedubail SP, Kini R. Assessment of the number of root canals in the maxillary and mandibular molars: A radiographic study using cone beam computed tomography. J Conserv Dent. 2017;20(5):288-92. do1:10.4103/jcd.jcd_73_17.

13. Reis A, Soares RG, Barletta FB, Fontanella VRC, Mahl CRW. Second Canal in Mesiobuccal Root of Maxillary Molars Is Correlated with Root Third and Patient Age: A Cone-beam Computed Tomographic Study. J Endod. 2013;39(5):588-92. [01:10.1016/1.joen.2013.01.003.

14. Lee JH, Kim KD, Lee JK, Park W, Jeong JS, Lee Y, et al. Mesiobuccal root canal anatomy of Korean maxillary first and second molars by cone-beam computed tomography. Oral Surg Oral Med Oral Pathol Oral Radiol Endod. 2011;111:785-91.

15. Fernandes NA, Herbst D, Postma TC, Bunn BK. The prevalence of second canals in the mesiobuccal root of maxillary molars: A cone beam computed tomography study. Aust Endod J. 2019;45(1):46-50. doi:10.1111/aej.12263

16. Guo J, Vahidnia A, Sedghizadeh P, Enciso R. Evaluation of Root and Canal Morphology of Maxillary Permanent First Molars in a North American Population by Cone-beam Computed Tomography. J Endod. 2014;40(5):635-9. doi:10.1016/j.joen.2014.02.004

\section{Author biography}

Altaf Hussain Chalkoo, Professor and HOD

Rayees Ahmad Sheikh, Postgraduate Student

Shamshad Begum, Senior Resident

Cite this article: Chalkoo AH, Sheikh RA, Begum S. Prevalence of mesiobuccal 2 canal in first maxillary molar: A retrospective radiographic study. IP Int J Maxillofac Imaging 2020;6(4):108-111. 